\title{
Role of kif2c, A Gene Related to ALL Relapse, in Embryonic Hematopoiesis in Zebrafish
}

\author{
Chang-Kyu Oh ${ }^{1, \dagger}{ }^{\text {, Ji Wan Kang }}{ }^{2,+}{ }^{+}$, Yoonsung Lee ${ }^{1}$, Kyungjae Myung ${ }^{1}$, Mihyang $\mathrm{Ha}^{2}$, \\ Junho Kang ${ }^{2}$, Eun Jung Kwon ${ }^{2}$, Youngjoo Kim ${ }^{2}$, Sae-Ock Oh ${ }^{3}$, Hye Jin Heo ${ }^{3}$, Shin Kim ${ }^{4,5, *(1)}$ \\ and Yun Hak Kim $3,6, *$ (D) \\ 1 Center for Genomic Integrity, Institute for Basic Science (IBS), Ulsan 44919, Korea; \\ ck1988@ibs.re.kr (C.-K.O.); yoonsunglee@ibs.re.kr (Y.L.); kmyung@ibs.re.kr (K.M.) \\ 2 Interdisciplinary Program of Genomic Science, Pusan National University, Yangsan 50612, Korea; \\ jwkang3929@naver.com (J.W.K.); mh2059389@naver.com (M.H.); rkdwnsgh2002@nate.com (J.K.); \\ sdb0419@naver.com (E.J.K.); asdoper0630@naver.com (Y.K.) \\ 3 Department of Anatomy, School of Medicine, Pusan National University, Yangsan 50612, Korea; \\ hedgehog@pusan.ac.kr (S.-O.O.); hjheo0303@gmail.com (H.J.H.) \\ 4 Department of Immunology, School of Medicine, Keimyung University, Dalseo-gu, Daegu 42601, Korea \\ 5 Institute of Medical Science, Keimyung University, Dalseo-gu, Daegu 42601, Korea \\ 6 Department of Biomedical Informatics, School of Medicine, Pusan National University, Yangsan 50612, Korea \\ * Correspondence: god98005@dsmc.or.kr (S.K.); yunhak10510@pusan.ac.kr (Y.H.K.); \\ Tel.: +82-53-258-7359 (S.K.); +82-51-510-8091 (Y.H.K.); Fax: +82-53-258-7355 (S.K.); +82-51-510-8049 (Y.H.K.) \\ + These authors contributed equally to this work.
}

Received: 6 March 2020; Accepted: 27 April 2020; Published: 28 April 2020

check for updates

\begin{abstract}
Relapse of acute lymphoblastic leukemia (ALL) is dangerous and it worsens the prognosis of patients; however, prognostic markers or therapeutic targets for ALL remain unknown. In the present study, using databases such as TARGET, GSE60926 and GSE28460, we determined that KIF2C and its binding partner, KIF18B are overexpressed in patients with relapsed ALL compared to that in patients diagnosed with ALL for the first time. As 50\% of the residues are exactly the same and the signature domain of KIF2C is highly conserved between human and zebrafish, we used zebrafish embryos as a model to investigate the function of kif $2 c$ in vivo. We determined that kif $2 c$ is necessary for lymphopoiesis in zebrafish embryos. Additionally, we observed that kif2c is not related to differentiation of HSCs; however, it is important for the maintenance of HSCs as it provides survival signals to HSCs. These results imply that the ALL relapse-related gene KIF2C is linked to the survival of HSCs. In conclusion, we suggest that KIF2C can serve as a novel therapeutic target for relapsed ALL.
\end{abstract}

Keywords: KIF2C; TARGET; GEO; ALL; zebrafish; hematopoiesis

\section{Introduction}

Acute lymphoblastic leukemia (ALL) is a common type of blood cancer, which presents a high number of undifferentiated lymphoid cells in the peripheral blood and bone marrow [1]. The symptoms of ALL include fever, fatigue and bleeding or enlargement of lymph nodes; ALL generally occurs in children aged 2-5 years [2,3]. ALL treatment includes chemotherapy, radiation therapy, biological therapy or immunotherapy; however, there is a chance of relapse in certain patients [4-6].

Approximately $20 \%$ of young patients with ALL suffer from a relapse and their prognosis is very poor $[7,8]$. The ratio of relapse is enhanced by a short duration of the first remission, bone marrow involvement, age greater than 10 years, unfavorable cytogenetics or Down syndrome [9-11]. 
Despite many attempts to study the genetic factors involved in the relapse of ALL, significant genetic factors remain unknown.

KIF2C is a member of the kinesin-like protein family and this protein family can bind to the microtubules to transport organelles and segregate chromosomes during cell division. According to recent studies, KIF2C is also involved in DNA repair [12,13]. As KIF2C is important for segregation of chromosomes and transport of organelles, many studies suggest that $K I F 2 C$ functions as an oncogene in many solid tumors [14]. KIF2C forms a complex with KIF18B, which plays a major role in microtubule plus-end depolymerizing activity in mitotic cells. The primary function of KIF18B may be to transport KIF2C along the microtubules $[15,16]$. However, there has been limited research on the role of KIF2C and its relationship to KIF18B in leukemia.

Since the hematological processes and lineages of zebrafish and humans are evolutionally conserved in vertebrates, many researchers have used zebrafish as an in vivo model for studies on hematopoiesis [17-20]. Among them, Park et al. investigated the function of the leukemia-related gene cobll1 using zebrafish embryos and reported that it is important for blast formation in chronic myeloid leukemia [18]. Because zebrafish embryos are transparent, whole-mount in situ hybridization (WISH) is mainly used for the observation of target gene expression in zebrafish embryos. Additionally, gene knockdown studies, which are important for investigating the function of genes, can be performed easily via administering morpholino injection in zebrafish $[18,21]$.

In the present study, we conducted various experiments to evaluate the recurrence of B-precursor ALL (B-ALL). The following datasets were analyzed to confirm the expression of KIF2C in patients with ALL_pediatric ALL-Phase II (TARGET, 2018), TARGET_paired sample consisting of data from only paired samples extracted from the TARGET cohort, GSE60926 and GSE28460. In addition, the differences in KIF2C expression between samples from first-diagnosed and relapsed patients were statistically analyzed and the correlation between KIF2C and KIF18B was confirmed. Subsequently, the function of $k i f 2 c$ was evaluated using zebrafish embryos.

\section{Results}

\subsection{KIF2C Expression Is Up-Regulated in Relapse Samples}

As shown in Table 1, no statistical differences were observed between TARGET, TARGET_paired sample and GSE60926 in terms of gender, age, mixed-lineage leukemia (MLL) status or race. The $t$-test indicated that the KIF2C expression level in the relapse sample was significantly higher than that in the first-diagnosis sample in all cohorts ( $p$-values: $1.977 \times 10^{-4}$ in GSE28460, $8.945 \times 10^{-5}$ in GSE60926, $1.497 \times 10^{-5}$ in TARGET_paired sample and $1.232 \times 10^{-11}$ in TARGET-ALL, Figure 1).
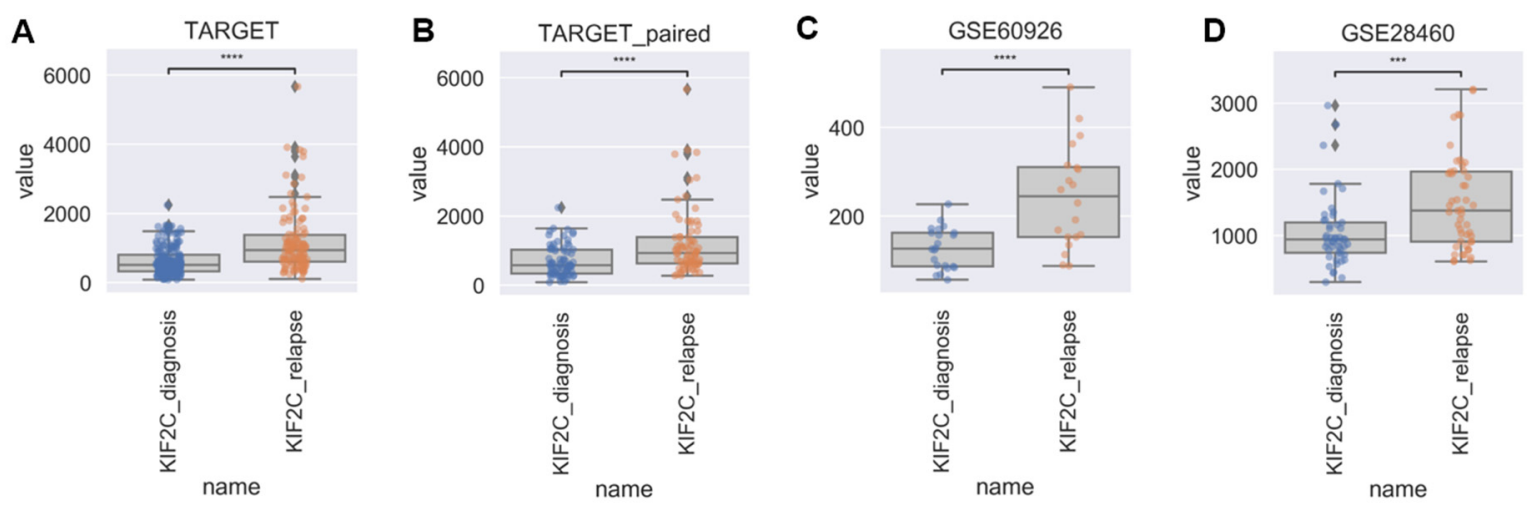

Figure 1. Differences in expression levels of KIF2C. Comparison of KIF2C expression levels in (A) TARGET, (B) TARGET_paired, (C) GSE60926, and (D) GSE28460 (**** indicates significance at $p$ value $<0.0001,^{* * *}$ indicates significance at $p$ value $\left.<0.001\right)$. 
Table 1. Patient characteristics in the four cohorts.

\begin{tabular}{|c|c|c|c|c|c|c|c|c|c|c|c|c|}
\hline \multirow{2}{*}{ Variables } & \multicolumn{3}{|c|}{$\begin{array}{c}\text { TARGET } \\
\text { (All Patients) }\end{array}$} & \multicolumn{3}{|c|}{$\begin{array}{c}\text { TARGET } \\
\text { (Paired Sample) }\end{array}$} & \multicolumn{3}{|c|}{ GSE60926 } & \multicolumn{3}{|c|}{ GSE28460 } \\
\hline & $\begin{array}{c}\text { Diagnosis } \\
(n=134)\end{array}$ & $\begin{array}{l}\text { Relapse } \\
(n=116)\end{array}$ & $p$ Value & $\begin{array}{c}\text { Diagnosis } \\
(n=76)\end{array}$ & $\begin{array}{l}\text { Relapse } \\
(n=76)\end{array}$ & $p$ Value & $\begin{array}{c}\text { Diagnosis } \\
(n=22)\end{array}$ & $\begin{array}{l}\text { Relapse } \\
(n=20)\end{array}$ & $p$ Value & $\begin{array}{c}\text { Diagnosis } \\
(n=49)\end{array}$ & $\begin{array}{l}\text { Relapse } \\
(n=49)\end{array}$ & $p$ Value \\
\hline \multicolumn{13}{|c|}{ Sex } \\
\hline Male & $74(55.2)$ & $64(55.2)$ & 1 & $42(55.3)$ & $42(55.3)$ & 1 & $15(68.2)$ & $14(70.0)$ & 0.878 & I & 1 & I \\
\hline Female & $60(44.8)$ & $52(44.8)$ & 1 & 34 (44.7) & 34 (44.7) & 1 & $6(27.3)$ & $5(25.0)$ & 0.75 & I & I & I \\
\hline \multicolumn{13}{|c|}{ Age } \\
\hline$<10$ & 79 (59.0) & 69 (59.5) & 0.963 & 44 (57.9) & 44 (57.9) & 1 & 16 (72.7) & $14(70.0)$ & 0.821 & I & 1 & I \\
\hline$\geq 10$ & $55(41.0)$ & 47 (40.5) & 0.955 & $32(42.1)$ & $32(42.1)$ & 1 & $6(27.3)$ & $6(30.0)$ & 0.721 & I & I & I \\
\hline \multicolumn{13}{|c|}{ MLL Status } \\
\hline Positive & $6(4.5)$ & $4(3.4)$ & 0.695 & $3(3.9)$ & $3(3.9)$ & 1 & I & I & I & I & I & I \\
\hline Negative & 105 (78.4) & $86(74.1)$ & 0.121 & $58(76.3)$ & $58(76.3)$ & 1 & I & I & I & I & I & I \\
\hline Unknown & $23(17.2)$ & $26(22.4)$ & 0.683 & 15 (19.7) & 15 (19.7) & 1 & I & I & I & I & 1 & I \\
\hline \multicolumn{13}{|c|}{ Race } \\
\hline White & 94 (70.1) & $81(69.8)$ & 0.979 & 54 (71.1) & 54 (71.1) & 1 & I & I & I & I & 1 & I \\
\hline Asian & $4(3.0)$ & $2(1.7)$ & 0.548 & $2(2.6)$ & $2(2.6)$ & 1 & I & I & I & I & I & I \\
\hline Black or African American & 15 (11.2) & $16(13.8)$ & 0.603 & $9(11.8)$ & $9(11.8)$ & 1 & l & l & l & l & l & l \\
\hline Etc. & $21(15.7)$ & $17(14.7)$ & 0.856 & $11(14.5)$ & $11(14.5)$ & 1 & l & l & l & / & 1 & / \\
\hline
\end{tabular}




\subsection{KIF2C and KIF18B Are Positively Correlated before and after Relapse}

Since KIF18B forms a complex with KIF2C, the difference in the expression level of KIF18B was analyzed in each B-ALL cohort. Similar to KIF2C, KIF18B was increased in relapsed samples compared to diagnosis in all cohorts (Figure 2). Next, Spearman correlation analysis was performed to investigate the correlation between KIF2C and KIF18B. As a result, all cohorts showed a strong correlation with a correlation coefficient of 0.7 or higher regardless of the diagnosis time and recurrence time (Figure S1).
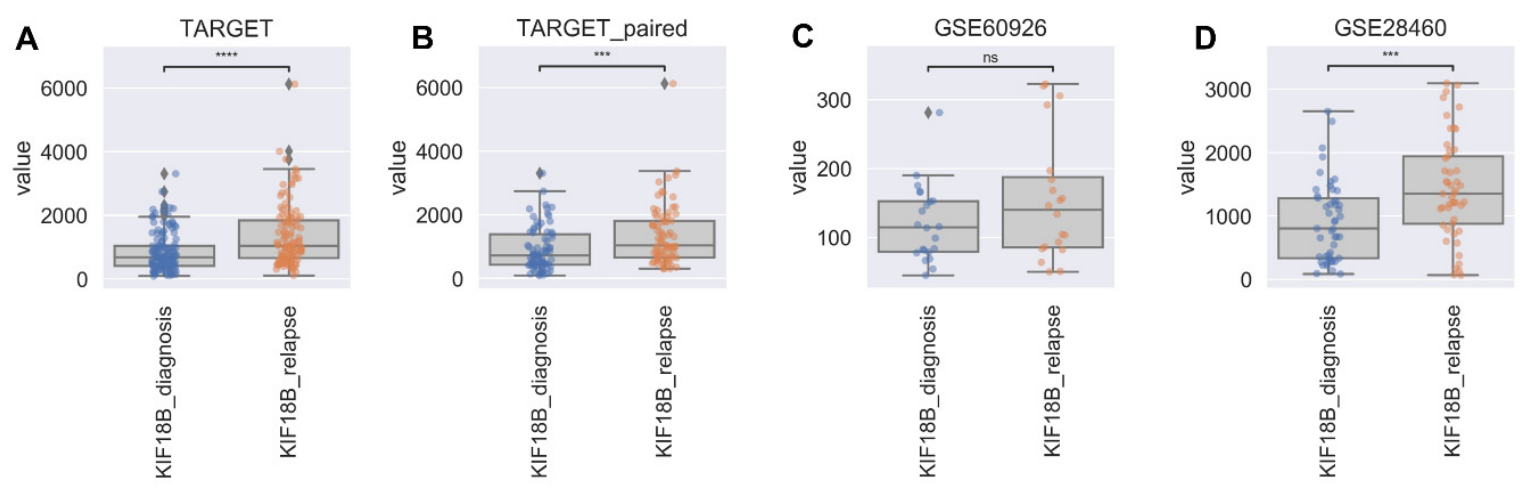

Figure 2. Differences in Expression Levels of KIF18B in B cell acute lymphoblastic leukemia (ALL). Comparison of KIF18B expression levels in (A) TARGET, (B) TARGET_paired sample, (C) GSE60926 and (D) GSE28460 ${ }^{* * * *}$ indicates significance at $p$ value $<0.00011^{* * *}$ indicates significance at $p$ value $<0.001$, ns means that there is no significant difference).

\section{3. kif2c Expression is Reduced by Morpholino Injection in Zebrafish Embryos}

According to the Ensemble database, the amino acid sequence of KIF2C is highly conserved between humans and zebrafish. Alignment between human KIF2C and zebrafish kif2c shows $50 \%$ of the residues to be exactly same, $15 \%$ structural similarity in the residues and $10 \%$ of gaps in compositional matrix adjustment (Figure S2). As KIF2C is important for the segregation of chromosomes during mitosis, the tubulin-binding site and the nucleotide-binding site are considered signature domains of KIF2C. We confirmed that these two sites are highly conserved between humans and zebrafish, indicating that zebrafish can function as a good in vivo model to investigate the functions of KIF2C (Figure S2). For the knockdown of kif2c in zebrafish, we used splice-blocking morpholino. To optimize the dosage of morpholino, we injected $2.5 \mathrm{ng}, 5 \mathrm{ng}$ and $10 \mathrm{ng}$ of kif2c-targeting morpholino at the 1-cell stage of the zebrafish embryos. Further, the mRNA expression of kif2c-targeting morpholino-injected embryos was compared with that of the control embryos. We observed that embryos injected with $10 \mathrm{ng}$ of morpholino showed reduced expression of kif2c, which was less than $50 \%$ compared to that in control embryos, according to RT-PCR analysis (Figure 3A). Next, the efficiency of the knock-down was confirmed by quantitative RT-PCR (qRT-PCR). Similar to the results from RT-PCR, the expression of kif2c showed an approximately $50 \%$ reduction, according to qRT-PCR (Figure 3B). These data suggest that $10 \mathrm{ng}$ of morpholino is required for kif2c knockdown in zebrafish embryos. 
A

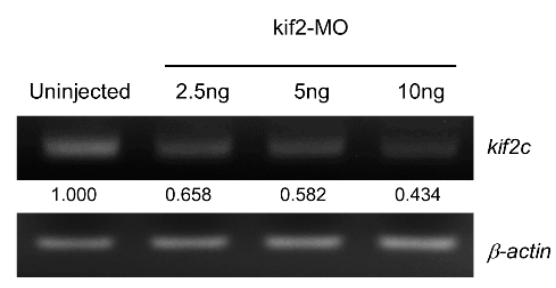

B

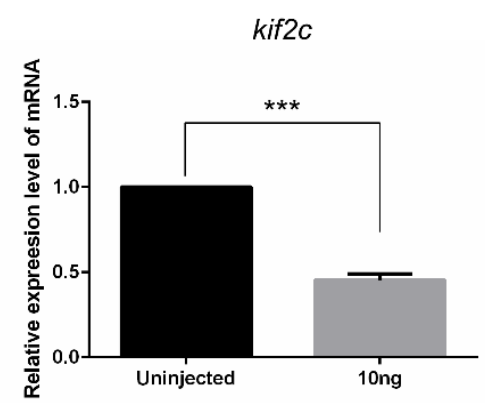

Figure 3. Expression of kif2c in zebrafish embryos is reduced by morpholino injection. (A) RT-PCR analysis of kif2c and $\beta$-actin using zebrafish embryos at 4 days post fertilization (dpf). Total RNA was isolated from uninjected embryos and kif2c-targeting morpholino-injected embryos (2.5 ng, $5 \mathrm{ng}$ and $10 \mathrm{ng}$ ). Relative expression of kif2c is quantified using ImageJ. (B) Quantitative RT-PCR analysis of kif2c. Relative mRNA expression of kif2c is compared between uninjected control embryos and kif2c-targeting morpholino injected embryos. (*** indicates significance at $p$ value $<0.001$ ).

\subsection{Lymphopoiesis Is Reduced in kif2c-Morphants}

We found that kif2c expression was higher in patients with relapsed B-ALL than in patients diagnosed with B-ALL for the first time (Figure 1). Similarly, we focused on elucidating the function of $k i f 2 c$ in hematopoiesis, especially in lymphopoiesis. The recombination activating gene ( $r a g$ ) is usually used as a lymphocyte-specific marker. Therefore, after knockdown of kif2c, the lymphocyte markers rag1 and rag 2 were analyzed using WISH and qPCR. Compared to the control embryos, kif2c-morphants showed reduced rag1-positive signal in the thymus of the zebrafish embryos (Figure 4A). A qPCR assay demonstrated that the relative mRNA expression of rag1 and rag2 was reduced in kif2c-morphants (Figure 4B). These data suggest that kif2c is important for lymphopoiesis.

A

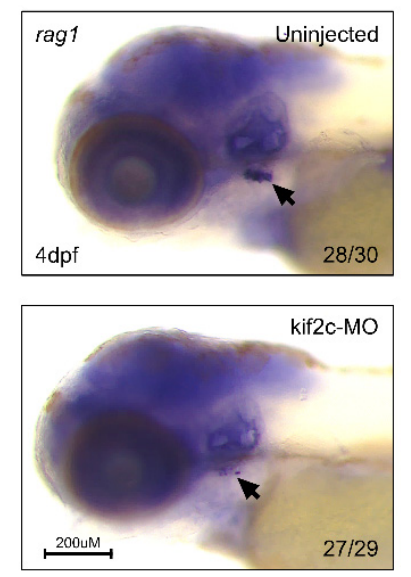

B

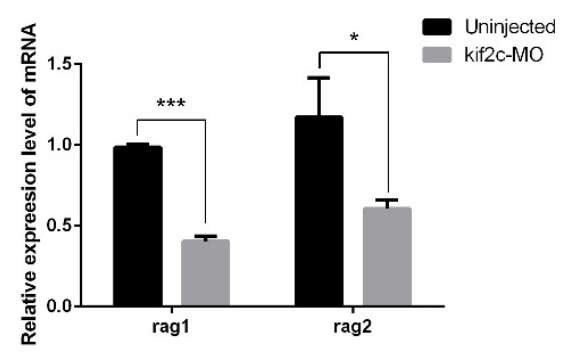

Figure 4. kif2c is linked to the development of lymphocytes in zebrafish embryos. (A) Whole-mount in situ hybridization of uninjected embryos and kif2c-targeting morpholino-injected embryos at 4 days post fertilization (dpf) using the lymphocyte marker rag1. Black arrows indicate the signal of rag1 at thymus. (B) qRT-PCR analysis of rag1 and rag2 expression in uninjected embryos and kif2c-targeting morpholino-injected embryos at $4 \mathrm{dpf}$. The expression of mRNA is normalized to that of $\beta$-actin mRNA levels (*** indicates significance at $p$ value $<0.001, *$ indicates significance at $p$ value $<0.05$ ).

\section{5. kif2c Is Necessary for Maintenance of Hematopoietic Stem Cells}

In the normal process of lymphopoiesis, hematopoietic stem cells (HSCs) are preferentially differentiated from endothelial cells of the dorsal aorta. During differentiation, HSCs show runx1 
expression at $28 \mathrm{~h}$ post fertilization (hpf) [22]. After differentiation, HSCs move to the caudal hematopoietic tissue (CHT) for expansion and these HSCs express $c m y b$ [23]. Thereafter, some of the HSCs are transported to the thymus for differentiation into lymphocytes [24].

As the lymphocytes were reduced in kif2c-morphants, we checked the stages of lymphopoiesis in kif2c-morphants to determine which stage of lymphopoeisis was inhibited. First, differentiation of HSCs was checked in the dorsal aorta using an HSC-specific probe, runx1, at $28 \mathrm{hpf}$. Similar to the control embryos, kif2c-morphants showed a normal signal of runx1 in the dorsal aorta (Figure 5A), suggesting that $k i f 2 c$ is not related to the differentiation of HSCs at initial stages. Further, HSCs at the CHT were checked using another HSC-specific probe, cmyb, at $72 \mathrm{hpf}$. In the normal process of hematopoiesis, cmyb-positive cells should expand in the CHT but kif2c-morphants showed a reduction in the number of $c m y b$-positive cells in the CHT (Figure 5B). These data suggest that kif $2 c$ is important for the maintenance of HSCs in CHT.

A
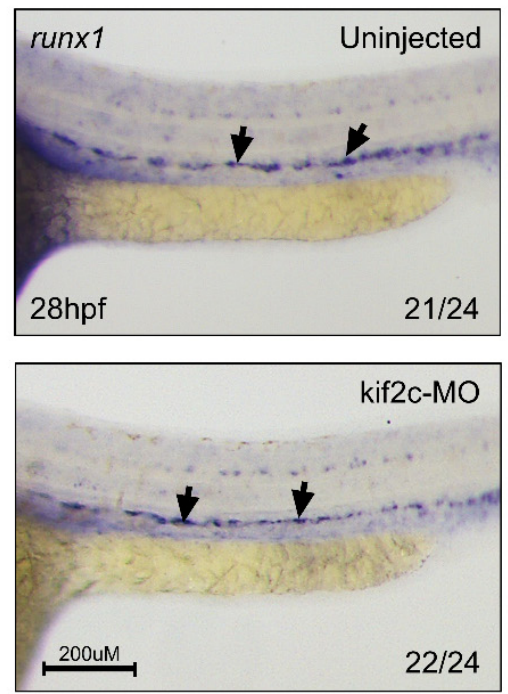

B
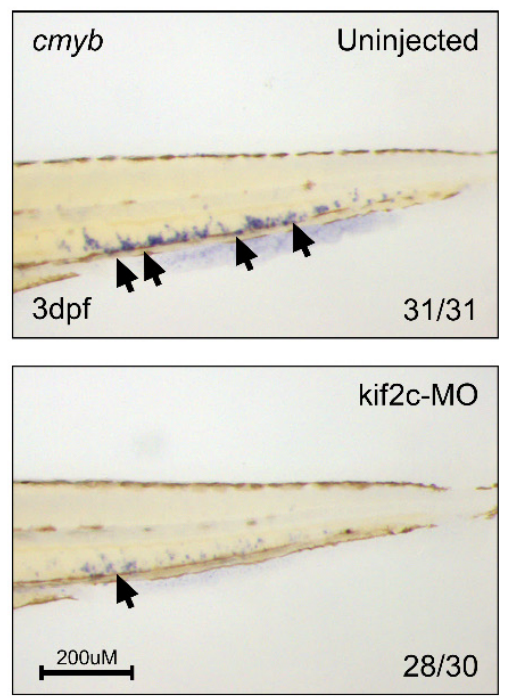

Figure 5. kif2c is related to the maintenance of hematopoietic stem cells. (A) Whole-mount in situ hybridization of uninjected embryos and kif2c-targeting morpholino-injected embryos at $28 \mathrm{hpf}$ using runx 1 probe. Black arrows indicate the signal of runx 1 at dorsal aorta. (B) Whole-mount in situ hybridization uninjected embryos and kif2c-targeting morpholino-injected embryos at 3 days post fertilization (dpf) using $c m y b$ probe. Black arrows indicate the signal of $c m y b$ at caudal hematopoietic tissue.

\section{6. kif2c Is Important for Survival of Hematopoietic Stem Cells}

To investigate the cause of reduction in HSCs in the CHT, we examined cell proliferation and apoptosis in kif2c-morphants. Cell proliferation in embryos was evaluated using the 5-ethynyl-2'deoxyuridine (EdU) assay at $3 \mathrm{dpf}$. Similar to that observed in normal embryos, the number of EdU-positive cells was not altered in the CHT of kif2c-morphants (Figure 6A), which indicates that cell proliferation is not related to reduced HSCs in the CHT. Next, acridine orange (AO) staining was used to detect apoptosis. Compared to the control embryos, the number of AO-positive cells was highly increased in the CHT of kif2c-morphants (Figure 6B), indicating that induced apoptosis is responsible for the reduction of HSCs in the CHT of kif2c-morphants. Since p53 is a key molecule inducing apoptosis [25], the expression of $p 53$ was analyzed to validate the apoptosis induced in kif $2 c$-morphants at $3 \mathrm{dpf}$. As AO-stained cells were increased in kif $2 c$-morphants, the expression of $p 53$ was also increased in the $\mathrm{CHT}$ (Figure $6 \mathrm{C}$ ). These data suggest that kif2c plays a role in the maintenance of HSCs by improving their survival. 
A
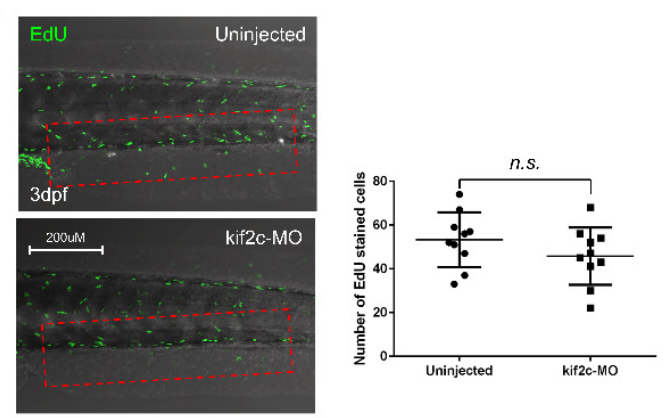

C

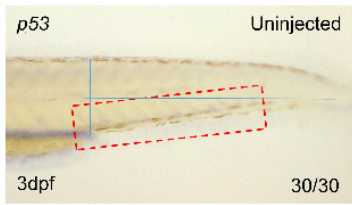

B
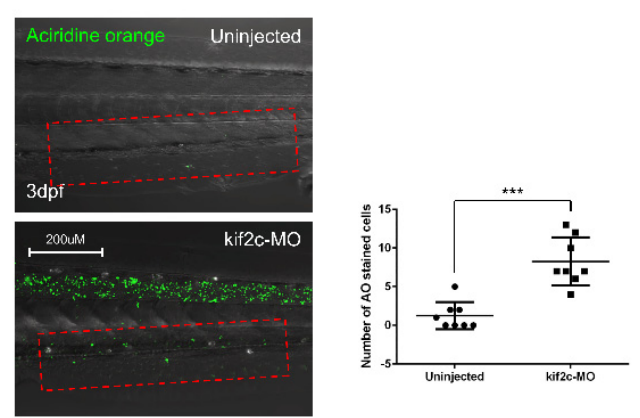

Figure 6. kif2c is important for survival of hematopoietic stem cells through regulation of DNA damage. (A) Lateral view of caudal hematopoietic tissue (CHT) using confocal microscope. Confocal imaging of 5-ethynyl-2'deoxyuridine (EdU)-stained control and kif2c-targeting morpholino-injected embryos. Right panel shows quantified data (n.s. indicates significance at $p$ value $>0.05$ ). Red frames indicate caudal hematopoietic tissue. (B) Apoptosis was observed by Acridine orange (AO) staining of control and kif2c-targeting morpholino-injected embryos. Right panel shows quantified data (*** indicates significance at $p$ value $<0.001)$. Red frames indicate caudal hematopoietic tissue. (C) Whole-mount in situ hybridization uninjected embryos and kif2c-targeting morpholino-injected embryos at 3 days post fertilization (dpf) using p53 probe. Red frames indicate caudal hematopoietic tissue.

\section{Discussion}

Relapse of ALL is dangerous and critical for the prognosis of patients; however, appropriate therapeutic target genes for ALL are not known. In the present study, through large data analysis, we found that KIF2C is significantly increased in relapsed B-ALL patients as compared to that in patients diagnosed with B-ALL for the first time. Additionally, it was confirmed that KIF2C and KIF18B showed high correlation before and after relapse. Next, using zebrafish embryos, we revealed that kif2c plays an important role in the maintenance of HSCs by improving their survival.

Various studies have suggested that KIF2C is important in solid tumors. TMA abdel-Fatah et al. suggested that KIF2C is a novel prognostic marker in breast cancer [26]. Gnjatic et al. reported that NY-CO-58/KIF2C is overexpressed in several solid tumors and that it induces frequent $\mathrm{T}$ cell response in colorectal cancer patients [27]. Gan et al. observed that KIF2C plays an oncogenic role in non-small cell lung cancer and that it can be negatively regulated by miR325-3p [13]. Although many studies show that KIF2C is an oncogenic factor in various solid tumors, we demonstrated for the first time that KIF2C plays an oncogenic role in leukemia.

KIF18B is known to play a role in the transport of KIF2C using the microtubules in complex with KIF2C $[15,16]$. On the basis of this information, we analyzed the correlation between KIF2C and KIF18B before and after relapse to determine if KIF18B shows the same tendency to increase as KIF2C. As a result, the strong correlation between KIF2C and KIF18B was confirmed and it can be predicted that the correlation between the two would play an important role in relapse.

We found that KIF2C is dramatically induced in relapsed B-ALL patients from different data bases and the zebrafish paralog, kif2c is important for the maintenance of HSCs by improving survival. Although leukemic hematopoiesis and normal hematopoiesis is different, our study suggests that KIF2C can be a critical gene in the relapse of ALL. Until now, relapsed ALL is treated using chemotherapy, radiotherapy or stem cell transplantation. Knowing that KIF2C is important in the relapse of ALL and for the maintenance of HSCs in zebrafish, we propose that KIF2C can be a novel therapeutic target 
for relapsed ALL. However, there are some limitations in this study. First, the lack of sufficient data may affect the credibility of the results. Second, since we identified only mRNA levels from databases and zebrafish experiments, it will be checked in protein level. Next, a more detailed study on the specific mechanism by which KIF2C induces survival signals for HSCs is required, using KIF2C as a therapeutic target for patients with ALL who suffer from relapse.

\section{Materials and Methods}

\subsection{Patients}

The gene expression data for pediatric B-cell ALL were downloaded from the cBioPortal for Cancer Genomics (https://www.cbioportal.org/) for TARGET-ALL and NCBI (National Center for Biotechnology Information) Gene Expression Omnibus (GEO, http://www.ncbi.nlm.nih.gov/geo/) database for GSE60926 and GSE28460 [28-32]. TARGET-ALL contributed to 1978 samples consisting of 134 diagnosis and 116 relapse bone marrow samples. Within the TARGET-ALL dataset, we additionally secured data representing the TARGET_paired sample cohort, which includes only paired samples; this dataset consists of 77 diagnosis and 77 relapse paired bone marrow samples. GSE60926 contributed to a total of 50 samples consisting of 22 diagnosis and 20 relapse bone marrow samples. GSE28460 contributed to 98 samples consisting of 49 diagnoses and 49 relapse paired bone marrow samples. The patient characteristics are described in Table 1.

\subsection{Statistical Methods for Analyzing ALL Databases}

The statistical differences in sex, age ( $<10$ or $\geq 10$ years), MLL status (positive, negative or unknown) and race (including White, Asian, Black or African American) were analyzed using the chi-square test. KIF2C expression levels in the first-diagnosis and relapse samples were compared using a $t$-test based on the statannot python package (statannot version 0.2.2 and python version 3.7.1, Python Software Foundation, 2020). Next, to examine the correlation between KIF2C and KIF18B, spearman correlation analysis was performed on the diagnosis samples and relapse samples of each cohort on the basis of the SciPy python package (SciPy version 1.4.1, Python Software Foundation, 2020).

\subsection{Zebrafish Maintenance and Morpholino Injection}

Wild-type zebrafish AB was maintained in an automatic circulation system (Genomic-Design) at $28.5^{\circ} \mathrm{C}$. All experiments using zebrafish embryos were performed in accordance with the guidelines of the Ulsan National Institute of Science and Technology (UNIST) Institutional Animal Care and Use Committee (IACUC) (IACUC approval number-UNISTIACUC-15-14, date: 2016-10-11). Zebrafish embryos were cultured using E3 solution in incubators at $28^{\circ} \mathrm{C}$. Splice-blocking morpholino targeting kif2c (Gene Tools, Philomath, OR, USA) was dissolved in DEPC water at $25 \mathrm{ng} / \mathrm{nL}$ stock solution. The sequence of kif2c-targeting morpholino is 5'-ACATTTAGTACAAACCTCTTTTCCT-3'. Morpholino targeting kif2c was injected in the embryos of wild-type zebrafish $\mathrm{AB}$ at the 1-cell stage of development. Microinjections were performed with a Femtojet 4i microinjector (Eppendorf, Hamburg, Germany).

\subsection{Quantitative RT-PCR}

Total RNA was isolated from 30 homogenized zebrafish embryos using $1 \mathrm{~mL}$ of TRIzol reagent (Molecular Research Center Inc., Cincinnati, OH, USA). Chloroform was added for protein separation and isopropanol was added for the precipitation of RNA. Reverse transcription of $3 \mu \mathrm{g}$ total RNA was performed using SuperScriptF IV Reverse transcriptase (Thermo Fisher, Waltham, Massachusetts, MA, USA). qRT-PCR analysis was performed using PowerUp SYBR green Master Mix (Thermo Fisher). Expression of target genes was analyzed by the comparative threshold method and the results were normalized to $\beta$-actin as an endogenous control gene. qRT-PCR data represents data obtained using biological and technical triplicates. 


\subsection{Whole-Mount In Situ Hybridization}

For WISH, the embryos were fixed using $4 \%$ paraformaldehyde in phosphate-buffered saline (PBS) and dehydrated using methanol at $-20^{\circ} \mathrm{C}$ overnight. Samples were incubated with acetone at $-20{ }^{\circ} \mathrm{C}$ for $30-40 \mathrm{~min}$. The samples were hybridized with a digoxigenin (DIG)-labeled antisense RNA probe in a hybridization buffer $(50 \%$ formamide, $5 \times \mathrm{SSC}, 500 \mu \mathrm{g} / \mathrm{mL}$ Torula yeast tRNA, $50 \mu \mathrm{g} / \mathrm{mL}$ heparin, $0.1 \%$ Tween-20 and $9 \mathrm{mM}$ citric acid (pH 6.5) for 3 days. The samples were then washed using $2 \times$ and $0.2 \times$ SSC solutions. The washed samples were blocked with normal goat serum and bovine serum albumin and incubated with alkaline phosphate-conjugated DIG antibodies (1:4000) (Roche, Mannheim, Germany) overnight at $4{ }^{\circ} \mathrm{C}$. The samples were then washed and incubated with alkaline phosphatase reaction buffer $[100 \mathrm{mM}$ Tris (pH 9.5), $50 \mathrm{mM} \mathrm{MgCl} 2,100 \mathrm{mM} \mathrm{NaCl}$ and 0.1\% Tween-20] and Alkaline phosphatase detection kit (NBT (p-nitroblue tetrazolium chloride)/BCIP (5-bromo-4-chloro-3-indolyl phosphate)) (Promega) for visualization of the WISH signal.

\subsection{Acridine Orange Staining of Zebrafish Embryos}

Zebrafish embryos were incubated with $50 \mu \mathrm{g} / \mathrm{mL}$ of AO solution in a dark environment for $1 \mathrm{~h}$ at room temperature. After $1 \mathrm{~h}$, the zebrafish embryos were washed thrice with E3 buffer, anesthetized using tricaine and observed under a confocal microscope (LSM880, Carl Zeiss, Jena, Germany).

\subsection{5-Ethynyl-2'deoxyuridine Assay}

Zebrafish embryos were saturated in $10 \mathrm{mM}$ EdU in E3 buffer with 15\% DMSO for $10 \mathrm{~min}$. The embryos were fixed using $4 \%$ paraformaldehyde in PBS. The fixed embryos were dehydrated in methanol at $-20{ }^{\circ} \mathrm{C}$. Next, the embryos were rehydrated in PBS with $0.1 \%$ Tween-20 and penetrated with $1 \%$ Triton $\mathrm{X}-100$ for $1 \mathrm{~h}$ at room temperature. The EdU signal was detected using the Click-iTEdU Alexa Fluor 488 Imaging Kit (Invitrogen) and the samples were imaged using an LSM880 confocal microscope (Carl Zeiss).

\subsection{Statistical Analysis in Zebrafish Experiments}

Statistical analysis was performed using Student's t-test. All experiments were performed in triplicates. Figures and graphs show the average of three independent experiments. The error bars indicate the standard error of the mean (SEM). A $p$-value less than 0.05 was considered statistically significant.

\section{Conclusions}

The main purpose of our study was to strengthen the foundation of precision medicine by analyzing large genome data. There is a growing need to discover novel therapeutic target genes for relapsed ALL. We analyzed the ability of KIF2C to serve as a therapeutic target of relapsed ALL and confirmed that KIF2C is highly induced in relapsed ALL patients; kif2c plays an important role in the homeostasis of HSCs through improvement of their survival. Based on our study, we propose that KIF2C is a novel therapeutic target of relapsed ALL.

Supplementary Materials: Supplementary materials can be found at http://www.mdpi.com/1422-0067/21/9/3127/ s1. Figure S1. Correlation between KIF2C and KIF18B in (A) the diagnosis sample of the TARGET cohort, (B) the relapse sample of the TARGET cohort, $(\mathrm{C})$ the diagnosis sample of the TARGET_paired sample cohort, the relapse sample of the TARGET_paired sample cohort, (E) the diagnosis sample of the GSE60926 cohort, (F) the relapse sample of the GSE60926 cohort, $(G)$ the diagnosis sample of the GSE28460 cohort, and (H) the relapse sample of the GSE28460 cohort. Figure S2. KIF2C is evolutionally conserved in vertebrates. Amino-acid sequence alignment of human KIF2C (query) and zebrafish kif2c (subject) using Pubmed.

Author Contributions: Conceptualization, C.-K.O., J.W.K., S.K. and Y.H.K.; Methodology, C.-K.O., J.W.K. and Y.K.; Formal analysis, J.W.K., K.M., S.-O.O., Y.L. and M.H.; Data Curation, J.K., E.J.K. and H.J.H.; Writing-original draft, C.-K.O. and J.W.K.; Writing-review \& editing, S.K. and Y.H.K.; Supervision, S.K. and Y.H.K.; Project Administration, S.K. and Y.H.K.; Funding Acquisition; K.M., S.K. and Y.H.K. All authors have read and agreed to the published version of the manuscript. 
Funding: This work was supported by grants from the Medical Research Center (MRC) Program and the Basic Science Research Program through the National Research Foundation of Korea (NRF) grant funded by the Korea government (NRF-2018R1A5A2023879, and 2014R1A5A2010008) and by the Institute for Basic Science (IBS-R022-D1).

Conflicts of Interest: The authors declare no conflict of interest.

\section{Abbreviations}

ALL Acute lymphoblastic leukemia

CHT Caudal hematopoietic tissue

DIG Digoxigenin

Dpf Days post fertilization

EdU 5-Ethynyl-2'deoxyuridine

GEO Gene Expression Omnibus

Hpf Hours post fertilization

HSCs Hematopoietic stem cells

MLL Mixed-lineage leukemia

Rag Recombination activating gene

WISH Whole-mount in situ hybridization

\section{References}

1. Ugolini, C.; Basolo, F.; Proietti, A.; Vitti, P.; Elisei, R.; Miccoli, P.; Toniolo, A. Lymphocyte and immature dendritic cell infiltrates in differentiated, poorly differentiated and undifferentiated thyroid carcinoma. Thyroid 2007, 17, 389-393. [CrossRef] [PubMed]

2. Jonsson, O.G.; Sartain, P.; Ducore, J.M.; Buchanan, G.R. Bone pain as an initial symptom of childhood acute lymphoblastic leukemia: Association with nearly normal hematologic indexes. J. Pediatr. 1990, 117 Pt 1, 233-237. [CrossRef]

3. Meir, H.M.; A Balawi, I.; Meer, H.M.; Nayel, H.; Al-Mobarak, M.F. Fever and granulocytopenia in children with acute lymphoblastic leukemia under induction therapy. Saudi Med. J. 2001, 22, 423-427. [PubMed]

4. Brentjens, R.J.; Davila, M.L.; Riviere, I.; Park, J.; Wang, X.; Cowell, L.G.; Bartido, S.; Stefanski, J.; Taylor, C.; Olszewska, M.; et al. CD19-targeted T cells rapidly induce molecular remissions in adults with chemotherapy-refractory acute lymphoblastic leukemia. Sci. Transl. Med. 2013, 5, 177ra38. [CrossRef] [PubMed]

5. Spiegler, B.; Kennedy, K.; Maze, R.; Greenberg, M.L.; Weitzman, S.; Hitzler, J.; Nathan, P.C. Comparison of long-term neurocognitive outcomes in young children with acute lymphoblastic leukemia treated with cranial radiation or high-dose or very high-dose intravenous methotrexate. J. Clin. Oncol. 2006, 24, 3858-3864. [CrossRef]

6. Einsiedel, H.G.; Von Stackelberg, A.; Hartmann, R.; Fengler, R.; Schrappe, M.; Janka-Schaub, G.; Mann, G.; Hählen, K.; Göbel, U.; Klingebiel, T.; et al. Long-term outcome in children with relapsed ALL by risk-stratified salvage therapy: Results of trial acute lymphoblastic leukemia-relapse study of the Berlin-Frankfurt-Munster Group 87. J. Clin. Oncol. 2005, 23, 7942-7950. [CrossRef]

7. Locatelli, F.; Schrappe, M.; Bernardo, M.E.; Rutella, S. How I treat relapsed childhood acute lymphoblastic leukemia. Blood 2012, 120, 2807-2816. [CrossRef] [PubMed]

8. Oriol, A.; Vives, S.; Hernández-Rivas, J.M.; Tormo, M.; Heras, I.; Rivas, C.; Bethencourt, C.; Moscardó, F.; Bueno, J.; Grande, C.; et al. Outcome after relapse of acute lymphoblastic leukemia in adult patients included in four consecutive risk-adapted trials by the PETHEMA Study Group. Haematologica 2010, 95, 589-596. [CrossRef]

9. Dördelmann, M.; Schrappe, M.; Reiter, A.; Zimmermann, M.; Graf, N.; Schott, G.; Lampert, F.; Harbott, J.; Niemeyer, C.; Ritter, J.; et al. Down's syndrome in childhood acute lymphoblastic leukemia: Clinical characteristics and treatment outcome in four consecutive BFM trials. Leukemia 1998, 12, 645-651. [CrossRef]

10. Hagedorn, N.; Acquaviva, C.; Fronkova, E.; Von Stackelberg, A.; Barth, A.; Stadt, U.Z.; Schrauder, A.; Trka, J.; Gaspar, N.; Seeger, K.; et al. Submicroscopic bone marrow involvement in isolated extramedullary relapses in childhood acute lymphoblastic leukemia: A more precise definition of "isolated" and its possible clinical implications, a collaborative study of the Resistant Disease Committee of the International BFM study group. Blood 2007, 110, 4022-4029. 
11. Heerema, N.; Palmer, C.G.; Weetman, R.; Bertolone, S. Cytogenetic analysis in relapsed childhood acute lymphoblastic leukemia. Leukemia 1992, 6, 185-192. [PubMed]

12. Zhu, S.; Paydar, M.; Wang, F.; Li, Y.; Wang, L.; Barrette, B.; Bessho, T.; Kwok, B.H.; Peng, A. Kinesin Kif2C in regulation of DNA double strand break dynamics and repair. Elife 2020, 9, 9. [CrossRef] [PubMed]

13. Gan, H.; Lin, L.; Hu, N.; Yang, Y.; Gao, Y.; Pei, Y.; Chen, K.; Sun, B. KIF2C exerts an oncogenic role in nonsmall cell lung cancer and is negatively regulated by miR-325-3p. Cell Biochem. Funct. 2019, 37, 424-431. [CrossRef] [PubMed]

14. Bie, L.; Zhao, G.; Wang, Y.-P.; Zhang, B. Kinesin family member 2C (KIF2C/MCAK) is a novel marker for prognosis in human gliomas. Clin. Neurol. Neurosurg. 2012, 114, 356-360. [CrossRef] [PubMed]

15. Tanenbaum, M.E.; Macurek, L.; Van Der Vaart, B.; Galli, M.; Akhmanova, A.; Medema, R.H. A complex of Kif18b and MCAK promotes microtubule depolymerization and is negatively regulated by Aurora kinases. Curr. Biol. 2011, 21, 1356-1365. [CrossRef]

16. Lee, Y.M.; Kim, E.; Park, M.; Moon, E.; Ahn, S.-M.; Kim, W.; Hwang, K.B.; Kim, Y.K.; Choi, W.; Kim, W. Cell cycle-regulated expression and subcellular localization of a kinesin-8 member human KIF18B. Gene 2010, 466, 16-25. [CrossRef]

17. Jing, L.; Zon, L.I. Zebrafish as a model for normal and malignant hematopoiesis. Dis. Models Mech. 2011, 4, 433-438. [CrossRef]

18. Han, S.H.; Kim, S.-H.; Lee, Y.; Choi, S.-Y.; Park, G.; Kim, D.-H.; Lee, A.; Kim, J.; Choi, J.-M.; Kim, Y.; et al. Cobll1 is linked to drug resistance and blastic transformation in chronic myeloid leukemia. Leukemia 2017, 31, 1532-1539. [CrossRef]

19. Gore, A.; Pillay, L.M.; Galanternik, M.V.; Weinstein, B.M. The zebrafish: A fintastic model for hematopoietic development and disease. Wiley Interdiscip. Rev. Dev. Biol. 2018, 7, e312. [CrossRef]

20. Zhu, X.; Liu, R.; Guan, J.; Zeng, W.; Yin, J.; Zhang, Y.C. Jak2a regulates erythroid and myeloid hematopoiesis during zebrafish embryogenesis. Int. J. Med. Sci. 2017, 14, 758-763. [CrossRef]

21. Palasin, K.; Uechi, T.; Yoshihama, M.; Srisowanna, N.; Choijookhuu, N.; Hishikawa, Y.; Kenmochi, N.; Chotigeat, W. Abnormal development of zebrafish after knockout and knockdown of ribosomal protein L10a. Sci. Rep. 2019, 9, 1-11. [CrossRef] [PubMed]

22. Perlin, J.R.; Robertson, A.L.; Zon, L.I. Efforts to enhance blood stem cell engraftment: Recent insights from zebrafish hematopoiesis. J. Exp. Med. 2017, 214, 2817-2827. [CrossRef] [PubMed]

23. Arulmozhivarman, G.; Krater, M.; Wobus, M.; Friedrichs, J.; Bejestani, E.P.; Müller, K.; Lambert, K.; Alexopoulou, D.; Dahl, A.; Stöter, M.; et al. Zebrafish In-Vivo Screening for Compounds Amplifying Hematopoietic Stem and Progenitor Cells: -Preclinical Validation in Human CD34+ Stem and Progenitor Cells. Sci. Rep. 2017, 7, 1-15. [CrossRef]

24. Rasighaemi, P.; Basheer, F.; Liongue, C.; Ward, A. Zebrafish as a model for leukemia and other hematopoietic disorders. J. Hematol. Oncol. 2015, 8, 29. [CrossRef]

25. Aubrey, B.J.; Kelly, G.L.; Janic, A.; Herold, M.J.; Strasser, A. How does p53 induce apoptosis and how does this relate to p53-mediated tumour suppression? Cell Death Differ. 2018, 25, 104-113. [CrossRef] [PubMed]

26. Abdel-Fatah, T.M.A.; McArdle, S.E.B.; Johnson, C.; Moseley, P.M.; Ball, G.R.; Pockley, A.G.; Ellis, I.O.; Rees, R.C.; Chan, S.Y.T. HAGE (DDX43) is a biomarker for poor prognosis and a predictor of chemotherapy response in breast cancer. Br. J. Cancer 2014, 110, 2450-2461. [CrossRef] [PubMed]

27. Gnjatic, S.; Cao, Y.; Reichelt, U.; Yekebas, E.F.; Nolker, C.; Marx, A.H.; Erbersdobler, A.; Nishikawa, H.; Hildebrandt, Y.; Bartels, K.; et al. NY-CO-58/KIF2C is overexpressed in a variety of solid tumors and induces frequent $\mathrm{T}$ cell responses in patients with colorectal cancer. Int. J. Cancer 2010, 127, 381-393. [CrossRef]

28. Hogan, L.E.; Meyer, J.A.; Yang, J.J.; Wang, J.; Wong, N.C.; Yang, W.; Condos, G.; Hunger, S.P.; Raetz, E.; Saffery, R.; et al. Integrated genomic analysis of relapsed childhood acute lymphoblastic leukemia reveals therapeutic strategies. Blood 2011, 118, 5218-5226. [CrossRef]

29. Ma, X.; Edmonson, M.; Yergeau, D.; Muzny, N.M.; Hampton, O.A.; Rusch, M.; Song, G.; Easton, J.; Harvey, R.C.; Wheeler, D.A.; et al. Rise and fall of subclones from diagnosis to relapse in pediatric B-acute lymphoblastic leukaemia. Nat. Commun. 2015, 6, 6604. [CrossRef]

30. Van Der Velden, V.H.J.; De Launaij, D.; De Vries, J.F.; De Haas, V.; Sonneveld, E.; Voerman, J.S.A.; De Bie, M.; Revesz, T.; Avigad, S.; Yeoh, A.E.J.; et al. New cellular markers at diagnosis are associated with isolated central nervous system relapse in paediatric B-cell precursor acute lymphoblastic leukaemia. Br. J. Haematol. 2016, 172, 769-781. [CrossRef] 
31. Cerami, E.; Gao, J.; Dogrusoz, U.; Gross, B.E.; Sumer, S.O.; Aksoy, B.A.; Skanderup, A.J.; Byrne, C.J.; Heuer, M.L.; Larsson, E.; et al. The cBio cancer genomics portal: An open platform for exploring multidimensional cancer genomics data. Cancer Discov. 2012, 2, 401-404. [CrossRef] [PubMed]

32. Gao, J.; Aksoy, B.A.; Dogrusoz, U.; Dresdner, G.; Gross, B.; Sumer, S.O.; Sun, Y.; Skanderup, A.J.; Sinha, R.; Larsson, E.; et al. Integrative analysis of complex cancer genomics and clinical profiles using the cBioPortal. Sci. Signal. 2013, 6, pl1. [CrossRef] [PubMed]

(C) 2020 by the authors. Licensee MDPI, Basel, Switzerland. This article is an open access article distributed under the terms and conditions of the Creative Commons Attribution (CC BY) license (http://creativecommons.org/licenses/by/4.0/). 